\title{
Resolution of psoriasis upon blockade of IL-15 biological activity in a xenograft mouse model
}

\author{
Louise S. Villadsen, ${ }^{1}$ Janine Schuurman, ${ }^{2}$ Frank Beurskens, ${ }^{2}$ Tomas N. Dam, ${ }^{3}$ \\ Frederik Dagnæs-Hansen, ${ }^{4}$ Lone Skov, ${ }^{1}$ Jørgen Rygaard, ${ }^{5}$ Marleen M. Voorhorst-Ogink, ${ }^{2}$ \\ Arnout F. Gerritsen, ${ }^{2}$ Marc A. van Dijk, ${ }^{2}$ Paul W.H.I. Parren, ${ }^{2}$ Ole Baadsgaard, ${ }^{6}$ \\ and Jan G.J. van de Winkel ${ }^{2,6,7}$
}

\author{
${ }^{1}$ Department of Dermatology, Gentofte University Hospital, Copenhagen, Denmark \\ ${ }^{2}$ Genmab B.V., Utrecht, The Netherlands \\ ${ }^{3}$ Department of Dermatology, Marselisborg University Hospital, Aarhus, Denmark \\ ${ }^{4}$ Department of Medical Microbiology, University of Aarhus, Aarhus, Denmark \\ ${ }^{5}$ Bartholin Institute, Copenhagen University Hospital, Copenhagen, Denmark \\ ${ }^{6} \mathrm{Genmab} \mathrm{A} / \mathrm{S}$, Copenhagen, Denmark \\ ${ }^{7}$ Department of Immunology, University Medical Center Utrecht, Utrecht, The Netherlands
}

\begin{abstract}
Psoriasis is a chronic inflammatory disease of the skin characterized by epidermal hyperplasia, dermal angiogenesis, infiltration of activated $T$ cells, and increased cytokine levels. One of these cytokines, IL-15, triggers inflammatory cell recruitment, angiogenesis, and production of other inflammatory cytokines, including IFN- $\gamma$, TNF- $\alpha$, and IL-17, which are all upregulated in psoriatic lesions. To investigate the role of IL-15 in psoriasis, we generated mAb's using human immunoglobulin-transgenic mice. One of the IL-15-specific antibodies we generated, 146B7, did not compete with IL-15 for binding to its receptor but potently interfered with the assembly of the IL-15 receptor $\alpha, \beta, \gamma$ complex. This antibody effectively blocked IL-15-induced T cell proliferation and monocyte TNF- $\alpha$ release in vitro. In a human psoriasis xenograft model, antibody $146 \mathrm{~B} 7$ reduced the severity of psoriasis, as measured by epidermal thickness, grade of parakeratosis, and numbers of inflammatory cells and cycling keratinocytes. These results obtained with this IL-15-specific mAb support an important role for IL-15 in the pathogenesis of psoriasis.
\end{abstract}

J. Clin. Invest. 112:1571-1580 (2003). doi:10.1172/JCI200318986.

\section{Introduction}

IL-15 is a proinflammatory cytokine of which the expression is upregulated under inflammatory conditions. This has been reported for monocytes stimulated with IFN- $\gamma$ and LPS or after infection with viruses, bacteria, or protozoa (1-3). IL-15 acts early in the inflammatory response and induces the production of other cytokines such as TNF- $\alpha$, IFN- $\gamma$, and IL-17 $(4,5)$. IL-15 furthermore amplifies inflammation by the recruitment and activation of T cells (4-6) and other inflammatory cells (7). IL-15 also induces angiogenesis (8),

Received for publication May 20, 2003, and accepted in revised form September 16, 2003.

Address correspondence to: Janine Schuurman, Genmab B.V., Jenalaan 18d, 3584 CK Utrecht, The Netherlands.

Phone: 31-30-2123-126; Fax: 31-30-2123-111;

E-mail: J.Schuurman@nl.genmab.com.

Louise S. Villadsen and Janine Schuurman contributed equally to this work.

Conflict of interest: This study has been financially supported by Genmab A/S (Copenhagen, Denmark). Genmab develops mAb 146B7 (HuMax-IL15) for immunotherapy of inflammatory diseases in close collaboration with Amgen (Thousand Oaks, California, USA).

Nonstandard abbreviations used: keyhole limpet hemocyanin (KLH); IL-15 receptor (IL-15R); room temperature (RT); antihuman IgG (anti-huIgG); propidium iodide (PI); Tris-buffered saline (TBS); cyclosporin A (CsA). plays a pivotal role in the development, survival, and function of NK cells (9-11), and has documented antiapoptotic effects $(12,13)$. These IL-15-induced effects have been suggested to play a pivotal role in disease pathogenesis of rheumatoid arthritis $(1,3-5,14)$ and may be important in other inflammatory diseases, like psoriasis, which has many similar features.

Psoriasis represents a chronic inflammatory skin disease affecting around $2 \%$ of the world's population. The histology of psoriasis is characterized by epidermal hyperplasia, dermal angiogenesis, and infiltration with mononuclear cells, including activated $\mathrm{T}$ cells and monocytes. The epidermal hyperplasia may be due to an increased number of cycling cells in the basal layer of the epidermis caused by a decrease in apoptosis or an increase in the rate of cell division. In accordance with this, psoriatic keratinocytes have been reported to be abnormally resistant to apoptosis (15-17). Several observations indicate a role for the immune system, especially activated $\mathrm{T}$ cells, in the pathogenesis of psoriasis (18-21). The $\mathrm{T}$ cells linked to the pathology of psoriasis primarily belong to the Th1 subtype $(22,23)$. In addition, also NK receptor-bearing cells have been found in psoriatic lesions $(24,25)$. Such cells have been demonstrated to induce full-fledged psoriatic plaques in the psoriasis xenotransplantation model in SCID mice, in which the local tissue immune response was 
dominated by IFN- $\gamma$ and IL-15 expression $(25,26)$. Several proinflammatory cytokines including IFN- $\gamma$ and TNF- $\alpha$ are upregulated in involved psoriatic skin, and the importance of TNF- $\alpha$ has been demonstrated by the clinical efficacy of TNF- $\alpha$ inhibitors (27-29). A significant upregulation of IL-15 and its receptor have been shown in psoriatic lesions $(12,30,31)$.

In this article we describe the generation of human antibodies directed against human IL-15. These antibodies were obtained using transgenic mice containing an unrearranged human antibody repertoire and in which endogenous murine antibody expression has been knocked out (32). Human IL-15-specific mAb's were prepared and selected based on in vitro characteristics. In a mouse xenograft model for psoriasis, using human psoriatic skin specimens, we tested the hypothesis that disregulation of IL-15 plays a key role in the pathogenesis of psoriasis. The resolution of psoriasis following the inhibition of IL-15 signaling strongly supports this hypothesis.

\section{Methods}

Immunization of buman immunoglobulin-transgenic mice. To obtain human mAb's against IL-15, human immunoglobulin transgenic mice strain $\mathrm{HCo} 12$ and HCo7 mice (Genmab, Utrecht, The Netherlands) were immunized with recombinant IL-15 (Immunex Corp., Seattle, Washington, USA) supplemented with either complete Freunds adjuvant (Difco, Detroit, Michigan, USA) or with incomplete Freunds adjuvant (Difco) subcutaneously, intraperitoneally (i.p.), or i.v. In several instances, IL-15 coupled to keyhole limpet hemocyanin (KLH) (Pierce Biotechnology, Rockford, Illinois, USA) was used for immunization. After several boosts with IL-15, sera of mice were tested for the presence of human antibodies directed against IL-15. To obtain hybridomas, mouse spleen and lymph node cells were fused with SP2/0 cells (33).

ELISA to determine binding of IL-15-specific antibodies to $I L-15$ and to mutant IL-15 proteins D8S and D8SQ108S. IL-15 or IL-15 mutant proteins D8SQ108S, or Q108S (kindly provided by Immunex Corp.; ref. 34) were coated onto ELISA plates. Residual binding sites were blocked by incubation with PBS containing $2 \%$ (vol/vol) chicken serum (PBSC, Life Technologies, Paisley, Scotland, United Kingdom) for 60 minutes. Serial dilutions of the IL-15-specific antibodies were incubated in PBSC, supplemented with $0.05 \%$ Tween20 (PBSTC), and detected with anti-human IgG (antihuIgG) Fc conjugated with horseradish peroxidase (Jackson, West Grove, Pennsylvania, USA; 1/5000 diluted in PBSTC). 2,2'-Azinobis-3-ethylbenzthiazoline-sulphonic-acid (ABTS) (Roche Diagnostics, Mannheim, Germany) was used as substrate according to the manufacturers' protocol.

IL-15 receptor $\alpha$ competition ELISA. IL-15 receptor $\alpha$ (IL-15R $\alpha$ ) (R\&D Systems, Minneapolis, Minnesota, USA) was coated onto ELISA plates. Following blocking and washing, IL-15 (50 $\mu$, room temperature [RT];
Immunex Corp.) was added for 10 minutes, followed by biotinylated antibody $(50 \mu \mathrm{l})$ in different concentrations (90 minutes, RT). Plates were incubated (60 minutes, RT) with streptavidin-poly-horseradish peroxidase (Sanquin, Amsterdam, The Netherlands) diluted 1:10,000 in PBSTC. ABTS was used as the substrate.

Culturing of PBMCs. Blood was obtained by venipuncture from healthy volunteers after informed consent. Purification of PBMCs was performed by density gradient centrifugation using Ficoll-Isopaque (Pharmacia, Uppsala, Sweden). PBMCs were cultured in RPMI 1640 medium (BioWhittaker, Verviers, Belgium) supplemented with penicillin $(50 \mathrm{U} / \mathrm{ml})$, streptomycin $(50$ $\mu \mathrm{g} / \mathrm{ml})$, L-glutamine $(2 \mathrm{mM})$ (BioWhittaker) and $10 \%$ FCS (Optimum C241, Multicell, Wisent Inc., St. Bruno, Quebec, Canada).

Binding of $146 B 7$ to IL-15-IL-15R complexes on Raji cells. Raji lymphoma cells (ATCC, Manassas, Virginia, USA) were preincubated with $10 \%$ human pooled $A B$ serum (Sanquin) in FACS buffer (PBS, 0.05\% BSA, and $0.02 \% \mathrm{NaNO}_{3}$ ) followed by incubation with IL-15 (diluted in FACS buffer with 10\% human $\mathrm{AB}$ serum) After washing, biotinylated Ab's (mAb 146B7 or huIgG1/א) (Sigma-Aldrich, Zwijndrecht, The Netherlands) were added. Bound Ab's were detected on a flowcytometer using streptavidin-phycoerythrin (DAKO, Glostrup, Denmark).

Inbibition of IL-15 and huIL-2-induced PBMC proliferation or TNF- $\alpha$ production by anti-IL-15 antibodies. PBMCs were cultured in triplicate in 96-well, U-bottom plates (Nalgene Nunc, Rochester, New York, USA), $1.5 \times 10^{5}$ cells per well in the presence or absence of different concentrations of huIL-2 (Chiron, Amsterdam, The Netherlands) or IL-15 (Immunex) and anti-IL-15 antibodies (mAb 146B7 or 404E4) or isotype control antibody in RPMI 1640 medium with $2 \mathrm{mM}$ L-glutamine, $50 \mathrm{IU} / \mathrm{ml}$ penicillin, $50 \mu \mathrm{g} / \mathrm{ml}$ streptomycin (Life Technologies), and 10\% heat-inactivated FCS (HyClone, South Logan, Utah, USA). Cells were incubated for 72 hours at $37^{\circ} \mathrm{C}$ and $5 \% \mathrm{CO}_{2}$. Proliferation was quantified using a colorimetric BrdU cell proliferation ELISA (Roche Diagnostics).

Apoptosis induction of PBMC by $m A b$ 146B7. PBMCs were cultured in 96-well, U-bottom plates (Nalgene Nunc), $1.5 \times 10^{5}$ cells per well in the presence or absence of $5 \mathrm{ng} / \mathrm{ml} \mathrm{IL-15}$ (Immunex) and $10 \mu \mathrm{g} / \mathrm{ml}$ anti-IL-15 antibody (mAb 146B7) or $10 \mu \mathrm{g} / \mathrm{ml}$ human isotype control antibody (mAb anti-KLH). Apoptotis was quantified after 5 days using an apoptosis detection kit (BD Biosciences, San Diego, California, USA). Staining of cells with the combination of annexin V-FITC and propidium iodide (PI) was performed according to the manufacturer's instructions for detection of live (annexin V-FITC neg/PIneg) cells, early apoptotic (annexin V-FITCpos/PIneg) and late apoptotic (annexin V-FITCpos/PIPos) cells. Specific cell markers were used to examine the apoptotic effect of mAb $146 \mathrm{~B} 7$ on specific cell populations. NK cells were selected by gating the $\mathrm{CD} 8{ }^{\text {pos }} \mathrm{CD} 56^{\text {pos }}$ cells by flow 
cytometry (using CD8 specific antibody (clone RPA-T8) and $\mathrm{CD}_{5} 6^{+}$(clone B159); T cells were selected by gating the CD3pos cells (using CD3 specific antibody, clone UCHT1); B cells were selected by gating the $\mathrm{CD} 20^{\text {pos }}$ cells by flow cytometry (using $\mathrm{CD} 20^{+}$specific antibody (clone 2H7); all antibodies used for flow cytometry were derived from BD Biosciences). Monocytes were selected based on forward scatter-side scatter. Data were analyzed by one-way ANOVA followed by post hoc Tukey's multiple comparison test. Analysis was performed using Graph Pad Prism (version 3.02 for Windows, Graph Pad Software, San Diego, California, USA).

Patients used for SCID mouse xenograft model. All patients had moderate to severe plaque psoriasis. The duration of disease was 21 to 43 years. None of the patients received any systemic treatment or phototherapy, but they were all considered for such treatment because of the severity of their disease. Two patients had received methotrexate, but the treatment was discontinued more than 7 years previously. All the patients had used topical treatment as steroids within the last month. Biopsies were taken from infiltrated red plaques located on the anterior or lateral aspect of the femoral region. Patients were encouraged to use moisturizers (Locobase, Yamanouchi Pharma, Leiderdorp, The Netherlands; Decubal, Sigma-Aldrich) for 1 to 2 days to remove scales from the biopsy site before the surgical procedure. Topical treatments were continued except for the anatomical region considered for biopsy. None of the patients received medication known to worsen psoriasis.

Human psoriasis SCID mouse xenograft model. The experimental protocol was approved by the local ethics committee and the Danish Experimental Animal Inspectorate. Keratome biopsies $(12 \times 5 \times 0.05 \mathrm{~cm}$ containing both dermis and epidermis) were obtained after informed consent from four psoriasis patients. The keratome biopsy from each patient was divided into 12 pieces of $1.5 \times 1.5 \mathrm{~cm}$ and transplanted onto the back of 12 C.B-17 SCID mice (2-3 months old, M \& B, Ry, Denmark). Three weeks after transplantation, mice were randomized into three groups (four mice each) for the following three treatments: i.p. injection of PBS (vehicle), $\mathrm{mAb} 146 \mathrm{~B} 7$ at a dose of $20 \mathrm{mg} / \mathrm{kg}$ on day 1 and $10 \mathrm{mg} / \mathrm{kg}$ on days 8 and 15, or CsA (positive control; Sandoz, Basel, Switzerland) at a dose of $10 \mathrm{mg} / \mathrm{kg}$ every second day for 15 days. One week after the last injection, mice were killed, and a 4-mm punch biopsy was taken from each xenograft. Biopsies were fixed in formalin for paraffin embedding, and stained in $\mathrm{H} \& \mathrm{E}$ (Merck, Darmstadt, Germany), and for Ki-67 nuclear antigen, $\mathrm{CD} 7^{+} \mathrm{NK}$ cells, $\mathrm{CD} 68^{+}$macrophages, and $\mathrm{CD}^{+}$or $\mathrm{CD}^{+} \mathrm{T}$ cells. The mice were kept under pathogen-free conditions throughout the study.

Clinical assessment. Prior to the first injection (baseline) and 1 week after the last injection, the severity of the psoriatic lesions was assessed for scaliness, induration, and erythema in a blinded fashion. The parameters were scored using the three-point scale: $0=$ complete lack of cutaneous involvement; 1 = slight involvement; 2 = moderate involvement; $3=$ severe involvement. On this scale from 0 to 3 , a maximal score of 3 represents severe scale, induration, and erythema of the psoriatic xenografts. The changes in clinical score of psoriasis were calculated by subtracting the end value from the value at baseline. The mean values for the mice in each treatment group were determined, and the data were summarized as mean \pm SEM. It should be noted that it is difficult to reach significant difference in these semiquantitative clinical assessments as discussed in detail previously by Dam et al. (35).

Immunostaining of biopsies from SCID mouse xenograft model. Deparaffinized sections were rinsed in distilled water. Different antigen-unmasking techniques were used. To unmask Ki-67, sections were placed in 0.01-M citrate buffer ( $\mathrm{pH}$ 6.0) and heated in a microwave set at $800 \mathrm{~W}$ for 7 minutes, followed by $560 \mathrm{~W}$ for $10 \mathrm{~min}$ utes. Sections were cooled at RT for 20 minutes and rinsed in Tris-buffered saline (TBS $0.1 \mathrm{M}, \mathrm{pH}$ 8.2). To unmask CD4 and CD8, sections were placed in $0.5-\mathrm{M}$ citrate buffer ( $\mathrm{pH} 10)$, and heated in a microwave as described above and rinsed in TBS $0.05 \mathrm{M}$ (pH 7.6). To unmask CD57 and CD68, sections were placed in $0.1 \%$ trypsin (Sigma-Aldrich) in $0.1 \% \mathrm{CaCl}_{2}\left(\mathrm{pH} 7.8,37^{\circ} \mathrm{C}\right)$ for $20 \mathrm{~min}$ and rinsed in water and TBS $0.05 \mathrm{M}(\mathrm{pH}$ 7.6). To stain for CD8, C8/144B (DAKO) was used diluted 1:100, followed by Envision/alkaline phosphatase (DAKO). The other sections were incubated with $10 \%$ (vol/vol) normal rabbit serum (DAKO) in TBS for 10 minutes and stained according to the ABC method using the following primary antibodies: MIB-1 (Ki-67; Immunotech, Marseille, France), NCL-NK1 (CD57, Novocastra Laboratories Ltd., Newcastle, United Kingdom), PG-M1 (CD68; DAKO), or NCL-CD4368 (CD4; Novocastra Laboratories), all diluted 1:50, then biotinylated rabbit anti-mouse antibody (DAKO) diluted 1:300 and streptABComplex/alkaline phosphatase (DAKO). For all the stainings, the color reactions were developed with Naphtol-AS-MX/Fast Red TR solution (Sigma-Alrdich), and Mayer's hematoxylin was used as counterstain. The negative controls consisted of omission of the primary antibody. As a positive control, a colon adenocarcinoma was used for the Ki-67 staining and a tonsil for the other stainings.

Histological evaluation. All sections were blinded prior to evaluation. The H\&E-stained sections were evaluated randomly by measurements for (a) epidermal thickness (in micrometers) measured from the stratum corneum to the deepest part of the rete pegs (b) number of inflammatory mononuclear cells per high-power field $(0.105 \times 0.135 \mathrm{~mm})$ in the upper dermis, and $(c)$ grade of parakeratosis rated on an arbitrary scale from 0 to 3 , where 0 was no parakeratosis, 1 was parakeratosis in less than one third of the section, 2 was parakeratosis in more than one third but less than two thirds of the section, and 3 was parakeratosis in more than two thirds of the section. The sections stained for Ki-67 
were evaluated for number of $\mathrm{Ki}-67^{+}$cycling keratinocytes-per-millimeter length of the section. The sections stained for CD57, CD68, CD4, and CD8 were evaluated for the number of positively stained cells-permillimeter length of the section.

The grade of parakeratosis was summarized as the percentage of mice in each treatment group that obtained scores $0,1,2$, or 3, respectively. For the other measurements, mean values for the mice in each treatment group were calculated, and the data were summarized as mean \pm SEM.

Statistical analysis of data obtained in the SCID mouse xenograft model. All tests are two sided. The significance level was set to 0.05 , and data from the two treatment groups of interest, $\mathrm{mAb} 146 \mathrm{~B} 7$ and PBS, were included in the analysis. ANOVA was used to test the null hypothesis of no difference between treatment groups for epidermal thickness, inflammatory cells, $\mathrm{Ki}-67^{+}, \mathrm{CD}^{+}$, and $\mathrm{CD}^{+}$cells. The analysis was adjusted for patient (as each patient donated skin to several mice) and mouse (as several observations were made for each mouse). Observations made for the same mouse were assumed to be correlated.
Observations made for different mice were assumed to be independent of each other. As only one observation is made for each mouse in the Ki-67, CD4, and CD8 data, no random factor is included in the analysis of these data. Fisher's Exact Test was used to test the null hypothesis of no association between the parakeratosis scores and treatment group. The nonparametric Kruskal-Wallis test was used to test for differences in clinical score, $\mathrm{CD} 57^{+}$, and $\mathrm{CD} 68^{+}+$cells between treatment groups.

\section{Results}

Generation of human antibodies specific for $I L-15$. To examine the role of IL-15 in the disease pathogenesis of psoriasis, human mAb's against IL-15 were generated using human immunoglobulin transgenic mice (32). These mice were immunized with IL-15, and hybridomas were obtained using conventional hybridoma technology (33). A panel of human mAb's against IL-15 was obtained that all recognized IL-15 as shown by ELISA and were of the IgG $1 / \mathrm{K}$ subclass. We selected two clones, mAb $146 \mathrm{~B} 7$ and mAb 404E4, for further evaluation. a

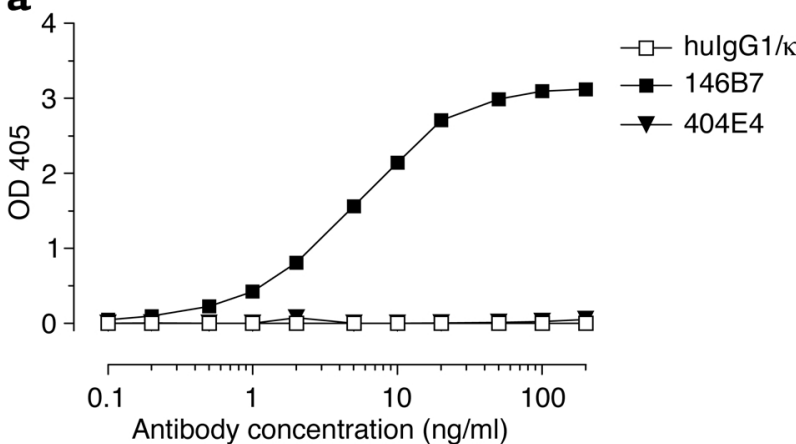

b

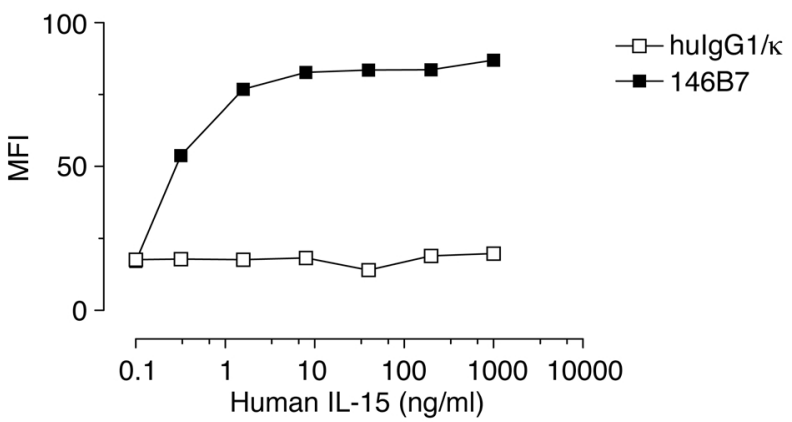

d

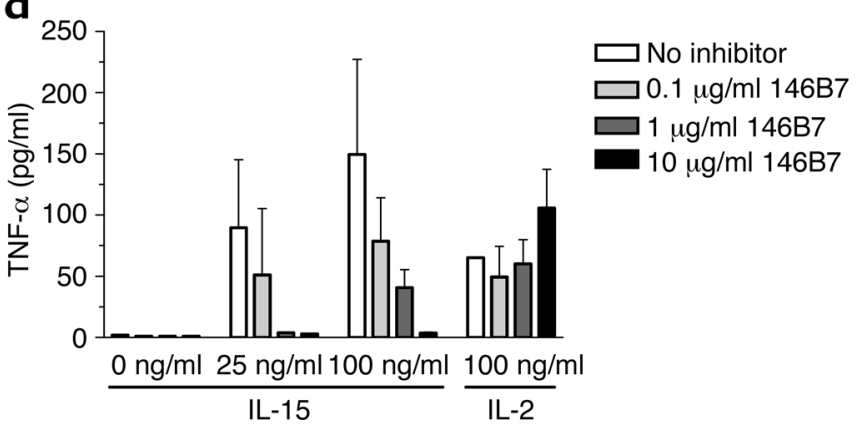

\section{Figure 1}

Recognition of receptor-bound IL-15 by mAb $146 \mathrm{~B} 7$ and inhibition of IL-15-induced effects. (a) Biotinylated mAb $146 \mathrm{~B} 7$ (filled squares) showed dose-dependent binding to IL-15 bound to IL-15R $\alpha$, which was coated onto an ELISA plate, whereas biotinylated 404E4 (filled triangles) did not show binding. Polyclonal hulgG1 (open squares) served as a control. This experiment was repeated two times, yielding similar results. (b) Dose-dependent binding of biotinylated mAb $146 \mathrm{~B} 7$ (filled squares) to IL-15 bound to Raji lymphoma cells expressing IL-15R $\alpha$ was shown by flow cytometry. Polyclonal hulgG1 (open squares) was used as a control. This experiment was repeated five times, yielding similar results. MFI, mean fluorescence intensity. (c) Effect of mAb $146 \mathrm{~B} 7$ on IL-15-induced proliferation. Human PBMCs were incubated with IL-15 $(12.5 \mathrm{ng} / \mathrm{ml})$ in combination with mAb 146B7 (filled squares), mAb 404E4 (filled inverted triangles), or with hulgG1 (open squares) for 72 hours. BrdU incorporation was measured to assay proliferation. Representative data of 9 individual experiments are shown. (d) $\mathrm{mAb} 146 \mathrm{~B} 7$ inhibits IL-15- but not IL-2-induced TNF- $\alpha$ production. Human PBMCs were incubated with IL-15 (0, 25, 100 ng/ml) or with IL-2 $(100 \mathrm{ng} / \mathrm{ml})$ in combination with mAb $146 \mathrm{~B} 7$ at various concentrations for 72 hours. The amounts of TNF- $\alpha$ produced were measured by ELISA. A representative experiment from a series of 11 experiments is shown. 

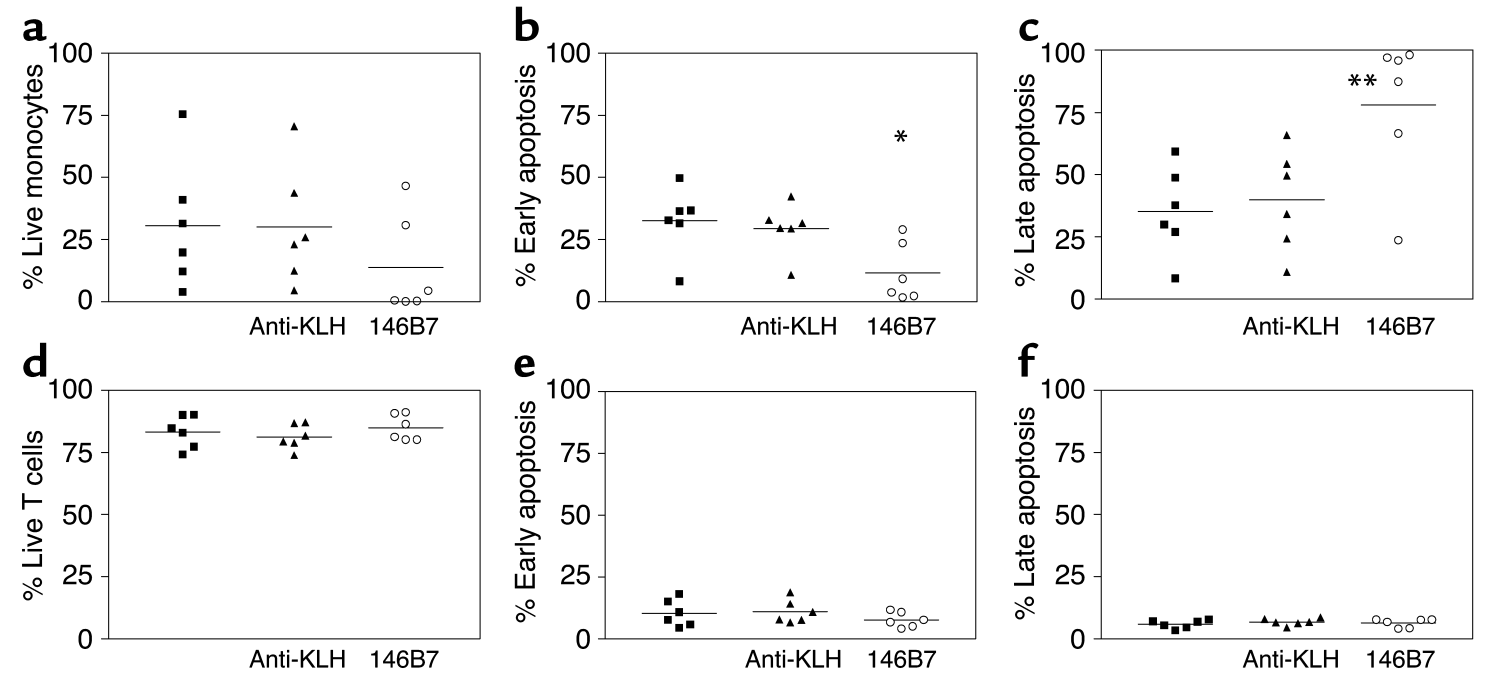

\section{Figure 2}

mAb 146B7 inhibits protection of apoptosis by IL-15. Human PBMCs were incubated with IL-15 (5 ng/ml) in culture medium alone (filled squares) or in combination with $10 \mu \mathrm{g} / \mathrm{ml} \mathrm{mAb} 146 \mathrm{~B} 7$ (open circles) or with $10 \mu \mathrm{g} / \mathrm{ml}$ isotype control antibody (filled triangles) for $5 \mathrm{days}$. Apoptosis was measured using annexin V conjugated with FITC and PI. Live (a and $\mathbf{d}$ ), early (b and $\mathbf{e})$, and late (c and $\mathbf{f})$ apoptotic cells were defined by annexin V-FITC negPIneg, annexin V-FITCPosPIneg, and annexin V-FITCPosPIpos staining by flow cytometry, respectively. Representative data of three individual experiments with six different PBMC donors are shown. (a-c) mAb $146 B 7$ inhibits IL-15-induced survival of monocytes. (d-f) No significant differences in comparison were found in the fraction of live and apoptotic T cells. ${ }^{*} P<0.05$, mAb $146 \mathrm{~B} 7$ versus no antibody treatment. ${ }^{*} P<0.005, \mathrm{mAb} 146 \mathrm{~B} 7$ versus no antibody treatment, and mAb $146 \mathrm{~B} 7$ versus anti-KLH.

Antibody $146 B 7$ recognizes receptor-bound IL-15. The characteristics of $\mathrm{mAb} 146 \mathrm{~B} 7$ and $\mathrm{mAb} 404 \mathrm{E} 4$, two representative antibodies of our panel, were examined in detail. First, we tested the ability of these IL-15-specific antibodies to compete with the IL-15R for binding to IL-15. The IL-15R present on T cells consists of a unique $\alpha$-chain, IL-15R $\alpha$, which has a very high affinity for IL-15: $\mathrm{K}_{\mathrm{D}} 10^{-11} \mathrm{M}$, and associates with the $\beta$-chain, and the $\gamma$-chain, through which signaling occurs $(2,36)$. The latter two chains are also present in the trimeric IL-2 receptor. In this test, the IL- $15 \mathrm{R} \alpha$ chain was coated onto ELISA plates, and after incubation with IL-15 increasing concentrations of antibodies were added (Figure 1a). mAb 404E4 did not bind to IL-15R $\alpha$-associated IL-15, indicating that $\mathrm{mAb} 404 \mathrm{E} 4$ recognizes an epitope involved in the interaction of IL-15 with the $\alpha$-chain of its receptor. The IL-15R $\alpha$ chain, in contrast, did not interfere with the binding of IL-15 to mAb 146B7, which indicates that $\mathrm{mAb} 146 \mathrm{~B} 7$ is a noncompeting antibody.

We examined the binding of $\mathrm{mAb} 146 \mathrm{~B} 7$ to the IL-15/ IL-15R complex in more detail using Raji cells expressing IL-15R $\alpha$ (37). Preincubating Raji cells with IL-15

\section{Figure 3}

Epitope specificity of human anti-IL-15 antibodies. mAb 146B7 (a) and mAb 404E4 (b) were tested for binding to human IL-15 (filled squares), IL-15 mutant Q108S (filled inverted triangles; Gln at residue 108 was replaced by Ser; a mutation in the $\gamma$-chain interaction site), and mutant D8SQ108S (filled triangles; Gln at residue 108 was replaced by Ser, and Asp at position 8 was substituted for Ser; mutations in both the $\beta$ and $\gamma$-chain interaction sites of IL-15 with its receptor) (34). resulted in a dose-dependent binding of $\mathrm{mAb} 146 \mathrm{~B} 7$ to these cells (Figure 1b). Also, preincubation of human monocytes with IL-15 facilitated a dose-dependent binding of mAb $146 \mathrm{~B} 7$ (data not shown; $n=3$ ).

$m A b 146 B 7$ inbibits T cell proliferation and TNF- $\alpha$ production induced by $I L-15$, but not IL-2. IL-15 triggers T cell proliferation as well as the induction of TNF- $\alpha$ production by monocytes. We assessed the efficacy of $\mathrm{mAb}$ $146 \mathrm{~B} 7$ and 404E4 on these IL-15-induced effects. Both $\mathrm{mAb} 404 \mathrm{E} 4$ and 146B7 inhibited IL-15-induced T cell proliferation (Figure 1c) if the antibodies were prein-

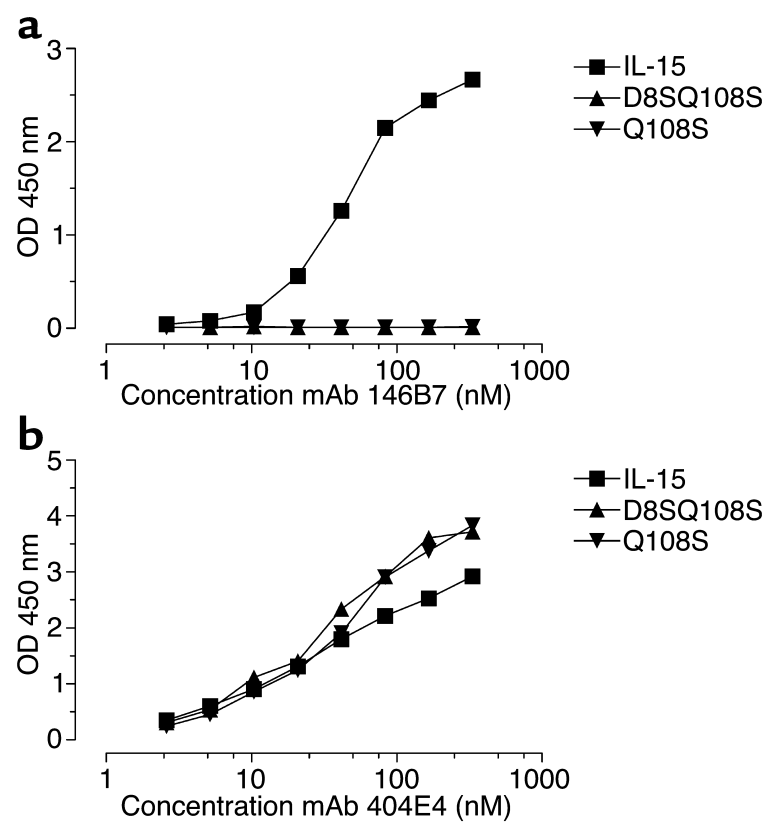



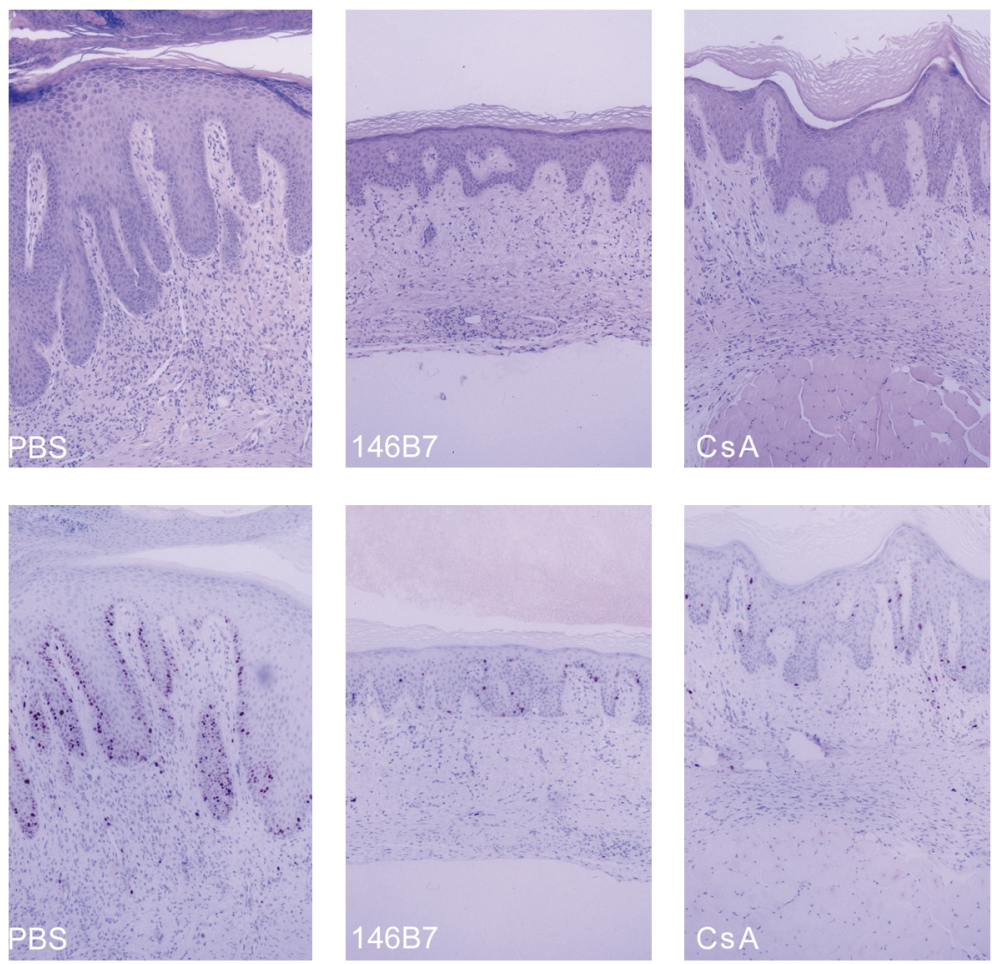

\section{Figure 4}

mAb 146B7 effectively alleviates psoriasis in a psoriasis SCID mouse xenograft model. SCID mice engrafted with human psoriatic skin were treated i.p. with PBS, $m A b$ $146 \mathrm{~B} 7$, or CsA. Skin grafts were harvested after treatment, embedded in paraffin, and stained with $\mathrm{H} \& \mathrm{E}$ (upper panels) or for Ki-67 nuclear antigen (lower panels). The tissue samples shown are from the same patient and are representative experiments with psoriatic skin obtained from four different patients.

cubated with IL-15 prior to addition to the cells. The noncompeting $\mathrm{mAb} 146 \mathrm{~B} 7$ proved noticeably more potent in inhibition of IL-15-induced proliferation (IC50 1 $\mu \mathrm{g} / \mathrm{ml}$ ) than $\mathrm{mAb} 404 \mathrm{E} 4$ (IC50 $\sim 7 \mathrm{~g} / \mathrm{ml}$ ). None of the IL-15-specific antibodies affected IL-2induced $\mathrm{T}$ cell proliferation (data not shown).

Next, we tested the effect of mAb $146 \mathrm{~B} 7$ on IL-15induced TNF- $\alpha$ production by PBMC. Antibody $146 \mathrm{~B} 7$ inhibited IL-15-induced TNF- $\alpha$ production in a dose-dependent manner, whereas no inhibition of
IL-2-mediated TNF- $\alpha$ production was observed (Figure 1d). In addition, IL-15induced IFN- $\gamma$ production was inhibited (data not shown; $n=3$ ).

$m A b 146 B 7$ induces apoptosis of monocytes in PBMC culture. To investigate which cell population was blocked by inhibiting IL-15-induced proliferation and TNF- $\alpha$ production by PBMC with $\mathrm{mAb} 146 \mathrm{~B} 7$, we investigated the induction of apoptosis. As shown in Figure 2a-c, monocytes were directly affected, and $\mathrm{mAb} 146 \mathrm{~B} 7$, in contrast to a control antibody against $\mathrm{KLH}$, strongly increased the fraction of apoptotic monocytes and reduced the fraction of live monocytes following 5 days of culture in the presence of IL- 15 . No significant differences in comparison were found in the fraction of live and apoptotic $T$ cells (Figure $2 \mathrm{~d}-\mathrm{f}$ ) and B cells (not shown); a small increase on cell death was seen on NK cells (not shown). It should be noted that the number of $T$ cells in PBMC culture in the presence of IL-15 strongly increased. However, as almost all $\mathrm{T}$ cells remained viable in the absence or presence of IL-15, no differences in the fraction of live or apoptotic cells are apparent.

Antibody $146 B 7$ epitope maps to IL-15 residues critical for $I L-15 R \quad \gamma$-chain binding. The most potent inhibition of IL-15mediated effects was observed for $\mathrm{mAb}$ 146B7, the antibody that is not interfering with IL-15 binding to IL-15R $\alpha$. We hypothesized mAb $146 \mathrm{~B} 7$ to interfere with assembly of the $\alpha \beta \gamma$ signaling complex of the receptor. To address this, we examined the binding epitope of mAb $146 \mathrm{~B} 7$ and 404E4 using IL-15 mutant proteins described by Pettit et al. (34). These mutants have substitutions in IL-15 sites that are important for either the $\gamma$-chain interaction or for both the $\beta$ - and $\gamma$-chain interaction of IL-15 (mutant Q108S: glutamine [Gln] at residue 108 was replaced by

Table 1

Reduction of psoriasis severity by anti-IL-15 treatment

\begin{tabular}{|c|c|c|c|c|}
\hline Treatment & $\begin{array}{l}\text { Epidermal thickness } \\
\qquad(\mu \mathrm{m}, \\
\text { mean } \pm \mathrm{SEM})\end{array}$ & $\begin{array}{c}\text { Inflammatory cells } \\
\text { (no./ high-power field, } \\
\text { mean } \pm \text { SEM) }\end{array}$ & $\begin{array}{c}\mathrm{Ki}-67^{+} \text {cells } \\
\text { (no./ mm section, } \\
\text { mean } \pm \mathrm{SEM} \text { ) }\end{array}$ & $\begin{array}{c}\text { Parakeratosis } \\
\text { (\% of mice with score } \\
0,1,2 \text {, and } 3 \text {, resp.) }\end{array}$ \\
\hline PBS & $380.6 \pm 43.9$ & $34.7 \pm 4.1$ & $189.8 \pm 33.7$ & $26.7,20.0,26.7,26.7$ \\
\hline $\mathrm{mAb} 146 \mathrm{~B} 7$ & $235.7 \pm 19.6^{A}$ & $21.0 \pm 3.0^{\mathrm{B}}$ & $76.7 \pm 9.7^{A}$ & $60.0,33.3,6.7,0.0^{\text {B }}$ \\
\hline CsA & $309.0 \pm 27.3$ & $28.2 \pm 4.1$ & $118.1 \pm 19.8$ & $25.0,37.5,18.8,18.8$ \\
\hline
\end{tabular}

mAb $146 \mathrm{~B} 7$ reduces psoriatic characteristics in a SCID mouse xenograft model. SCID mice engrafted with human psoriatic skin were treated i.p. with PBS $(n=15)$, mAb $146 \mathrm{~B} 7(n=15)$ or with CsA $(n=16)$. Biopsies were taken 1 week after stopping treatment and stained with H\&E or for Ki-67 and evaluated for psoriatic characteristics as epidermal thickness, number of inflammatory cells in upper dermis, and number of Ki-67 ${ }^{+}$cycling keratinocytes. Mean values for the mice in each treatment group were calculated, and the data were summarized as mean \pm SEM. The sections were also evaluated for the grade of parakeratosis, and the data were summarized as the percentage of mice in each treatment group that obtained the score 0 , 1 , 2, or 3 , respectively (resp.). Statistical differences between mAb 146B7- and the PBS-treated mice were calculated, and the effect of mAb $146 B 7$ was significant for all psoriatic characteristics $\left({ }^{A} P<0.005 ;{ }^{B} P<0.05\right)$. 


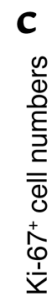

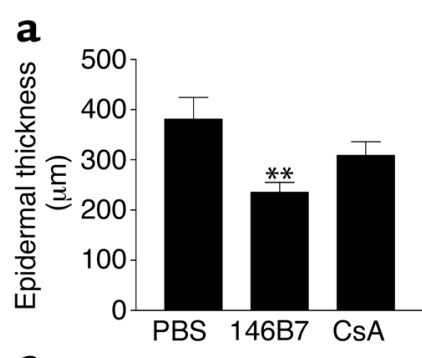

b
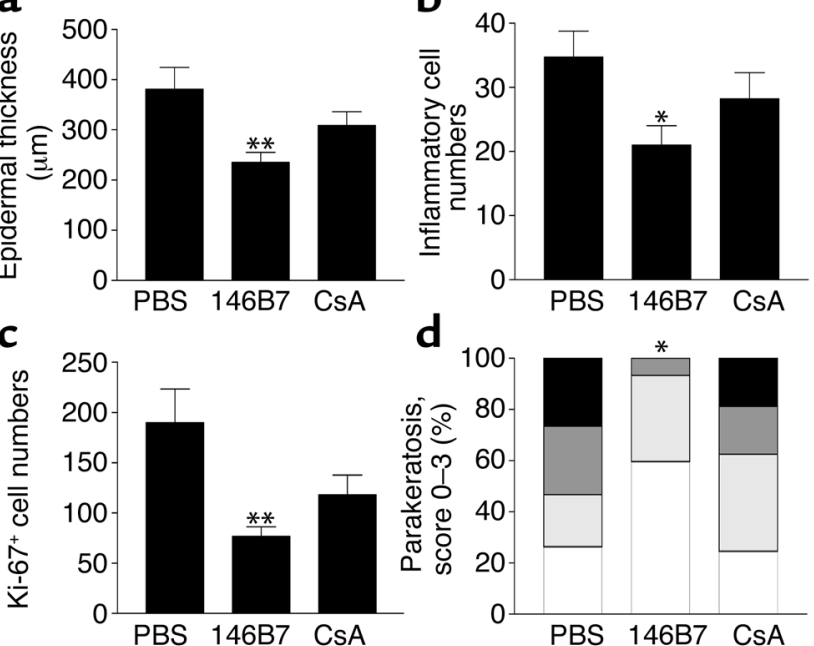

human IL-15 antibody (m112). A clear cytoplasmic staining of epidermal cells (keratinocytes) was observed with the IL-15-specific antibodies, whereas normal tissue showed a much lower intensity of IL-15 positivity (data not shown).

To examine the role of IL-15 in the pathophysiology of psoriasis, we tested the efficacy of the IL-15-specific antibody $\mathrm{mAb} 146 \mathrm{~B} 7$ in treatment of human psoriasis skin grafts transplanted onto SCID mice $(30,35$, 38,39 ). This model has previously been validated, and it has been shown that human psoriatic grafts can be successfully transplanted to SCID mice, retaining clinical, light microscopic, and immunohistochemical psoriatic skin characteristics when compared with pretransplanted skin (38).

A blinded clinical assessment of the skin grafts was performed prior to the first injection, and 1 week following the last injection and scored for severity of scaliness, induration, and erythema on a scale from 0 to 3 . A comparison of all four experiments conducted indicated that $\mathrm{mAb} 146 \mathrm{~B} 7$ treatment resulted in a reduction of the overall clinical score $(-1.1 \pm 0.2)$ of the xenografts compared with control mice treated with PBS $(-0.6 \pm 0.2 ; P=0.15)$.

Light microscopic characteristics of xenograft biopsies obtained from control mice, injected with PBS, indeed retained a characteristic psoriatic skin phenotype including hyperkeratosis, parakeratosis, acanthosis with elongated rete pegs, a conspicuous mononuclear cell infiltrate (Figure 4, upper panel), and an increased number of cycling keratinocytes measured as Ki- $67^{+}$keratinocytes (an indicator of keratinocyte proliferation) (Figure 4, lower panel). In contrast, xenografts from mice that were treated with $\mathrm{mAb} 146 \mathrm{~B} 7$ once weekly during 2 weeks resulted in near-complete resolution of psoriasis characteristics (Figure 4, upper panel), including normalization of the number of cycling keratinocytes (Figure 4, lower panel). Cyclosporin A (CsA), a potent antipsoriatic$$
\begin{aligned}
& a \\
& 0 \\
& 0 \\
& 0 \\
& 0 \\
& 0 \\
& \text { ह } \\
& 0 \\
& \hline 0 \\
& 0 \\
& 1 \\
& 0 \\
& 0
\end{aligned}
$$

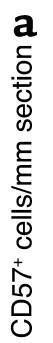

品
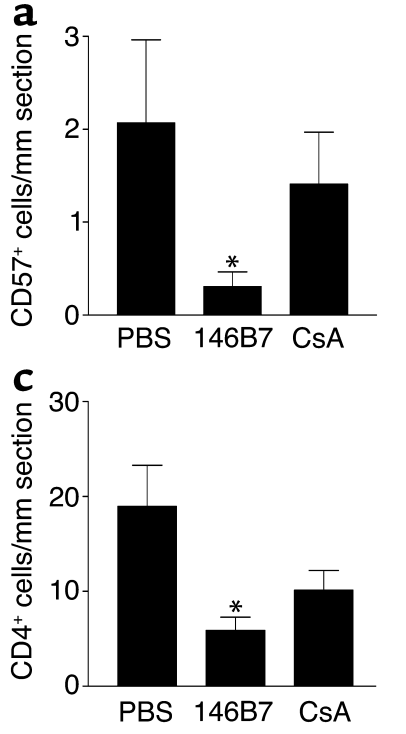
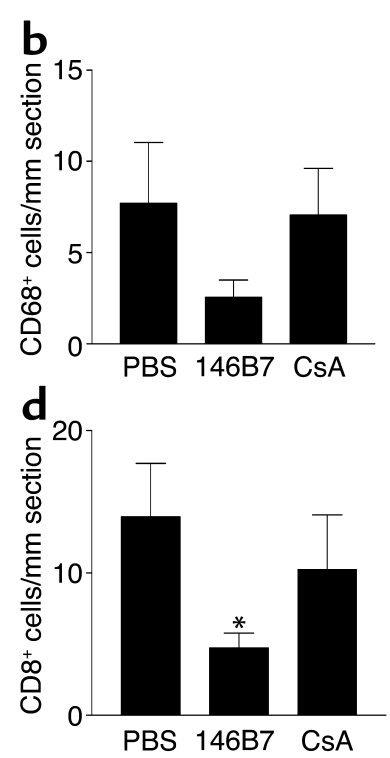

\section{Figure 6}

$\mathrm{mAb} 146 \mathrm{~B} 7$ results in a reduction in the number of $\mathrm{CD} 57^{+}, \mathrm{CD}^{\circ} 8^{+}$, $\mathrm{CD}^{+}$, and $\mathrm{CD}^{+}$cells in the psoriasis SCID xenograft model. SCID mice engrafted with human psoriatic skin were treated i.p. with PBS $(n=15)$, mAb 146B7 $(n=15)$, or with CsA $(n=16)$. Skin grafts were harvested after treatment, embedded in paraffin, and stained for CD57+ $\mathrm{NK}$ cells (a), CD68 ${ }^{+}$macrophages (b), CD4 ${ }^{+} \mathrm{T}$ cells $(\mathbf{c})$, and $\mathrm{CD} 8^{+} \mathrm{T}$ cells $(\mathbf{d})$, and evaluated for number of positively stained cells. Values shown represent mean \pm SEM. ${ }^{*} P<0.05$ (PBS compared with $\left.\mathrm{mAb} 146 \mathrm{~B} 7\right)$. 


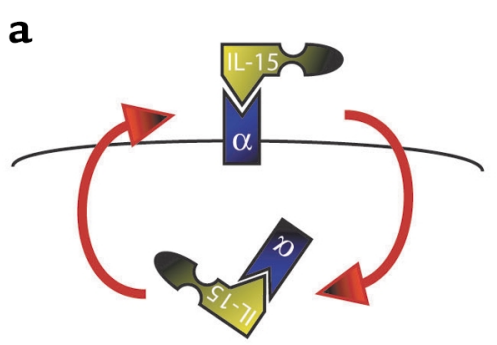

d

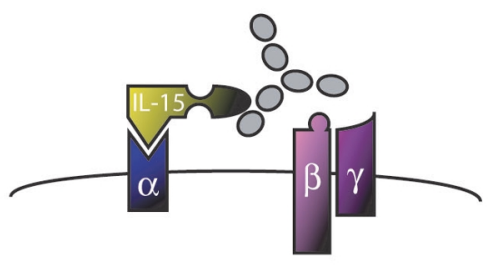

b

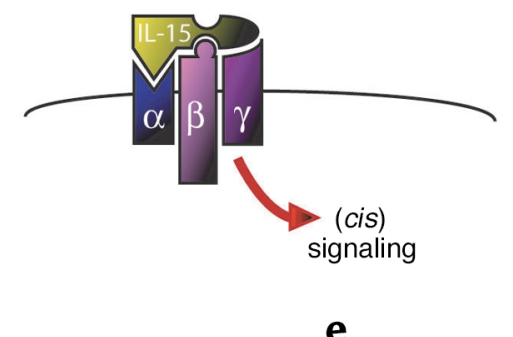

c

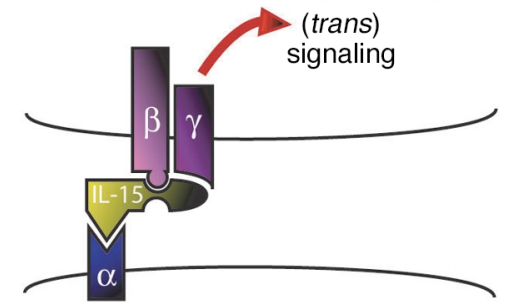

e

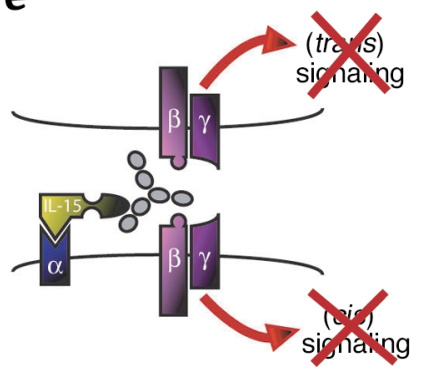

\section{Figure 7}

A recent paper by Dubois et al. (40) provided new insight in the mechanism of action of IL-15. The formation of IL-15/IL-15R $\alpha$ complexes on cell surfaces enables transendosomal recycling of IL-15, leading to persistence of surface-bound IL-15 (a). IL-15/IL-15R $\alpha$ complexes may induce signaling via IL-15R $\beta$ - and $\gamma$-chains expressed on the same cell in cis (b) or expressed on a distinct cell in trans (c). Antibody $146 \mathrm{~B} 7$ binds at the interaction site of IL-15 with the $\gamma$-chain $(\mathbf{d})$ and may effectively block signaling through the $\gamma$-chain both in cis and in trans (e).

drug with demonstrated efficacy in this model $(30,35$, $38,39)$, was included as a positive control. CsA treatment every second day during 2 weeks also resulted in attenuation of the psoriasis, although not to the same degree as mAb 146B7 (Figure 4) under these experimental conditions.

To quantify the immunohistochemical data shown in Figure 4, we measured epidermal thickness, the numbers of inflammatory cells in the upper dermis, the numbers of Ki- $67^{+}$cycling keratinocytes, and the grades of parakeratosis. mAb $146 \mathrm{~B} 7$ significantly reduced all four parameters compared to control mice (Table 1). First, mAb146B7 reduced the epidermal thickness measured from the stratum corneum to the deepest part of the rete pegs (Figure $5 \mathrm{a}):(235.7 \pm 19.6 \mu \mathrm{m}) \mathrm{com}$ pared with PBS $(380.6 \pm 43.9 \mu \mathrm{m})$ and CsA $(309.0 \pm 27.3$ $\mu \mathrm{m})$. Second, $\mathrm{mAb} 146 \mathrm{~B} 7$ reduced the number of inflammatory mononuclear cells in the upper dermis (21.0 \pm 3.0$)$, compared with PBS (34.7 \pm 4.1$)$, and CsA $(28.2 \pm 4.1)$ (Figure $5 \mathrm{~b})$ and the number of $\mathrm{Ki}-67^{+}$ cycling keratinocytes $(76.7 \pm 9.7)$ compared with PBS $(189.8 \pm 33.7)$, and CsA $(118.1 \pm 19.8)$ (Figure 5c). Finally, the grade of parakeratosis, an indicator of incomplete terminal differentiation of keratinocytes, was significantly reduced by $\mathrm{mAb} 146 \mathrm{~B} 7$ treatment (score 0: 60.0\%, 1: 33.3\%, 2: 6.7\%, and 3: 0.0\%) compared to PBS (score 0: 26.7\%, 1: 20.0\%, 2: 26.7\%, and 3: 26.7\%), and CsA (score 0:25.0\%, 1:37.5\%, 2: $18.8 \%$, and 3: 18.8\%) (Figure 5d).

In contrast, injection of mice with an isotype control antibody used at the same concentration as $\mathrm{mAb}$ 146B7 did not result in any decrease of any of the measured parameters (data not shown). To further analyze the reduction of the number of inflammatory cells, we analyzed cell types relevant to psoriasis and found that the number of CD57 $7^{+} \mathrm{NK}$ cells (Figure 6a), $\mathrm{CD}^{+} 8^{+}$macrophages (Figure 6b), and $\mathrm{CD}^{+}$(Figure $6 \mathrm{c}$ ) and $\mathrm{CD}^{+} \mathrm{T}$ cells (Figure $6 \mathrm{~d}$ ) were all reduced in the xenografts from the $\mathrm{mAb} 146 \mathrm{~B} 7$-treated mice (CD57: $0.3 \pm 0.2$, CD68: $2.6 \pm 0.9$, CD4: $5.9 \pm 1.4$, and CD8: $4.7 \pm 1.1)$ compared with the PBS-treated mice (CD57: $2.1 \pm 0.9$, CD68: $7.7 \pm 3.3$, CD4: $19.0 \pm 4.3$, and CD8: $14.0 \pm 3.7)$ and the CsA-treated mice (CD57: $1.4 \pm 0.6$, CD68: $7.1 \pm 2.6$, CD4: $10.1 \pm 2.1$, and CD8: $10.2 \pm 3.9$ ) (Figure 6). Except for $\mathrm{CD}^{2} 8^{+}$cells, the reductions induced by mAb $146 \mathrm{~B} 7$ compared with PBS were statistically significant for all measured parameters.

These experiments clearly showed that in SCID mice engrafted with human psoriatic skin, treatment with mAb $146 \mathrm{~B} 7$ mostly resolved psoriasis, as compared with treatment with PBS alone. Indeed, treatment with $\mathrm{mAb} 146 \mathrm{~B} 7$ resulted in a major reduction in epidermal thickness, in numbers of inflammatory cells and cycling keratinocytes, and in severity of parakeratosis in human skin grafts transplanted on to SCID mice.

\section{Discussion}

We generated a novel human antibody, mAb 146B7, which exhibits anti-inflammatory effects in vitro and in an in vivo psoriasis xenograft model. By using $\mathrm{mAb}$ $146 \mathrm{~B} 7$, we showed IL-15 to play a prominent role in the pathogenesis of psoriasis. Our data show mAb 146B7 to reduce the severity of psoriasis as measured by relevant disease parameters like epidermal thickness, number of inflammatory cells, parakeratosis, and cycling keratinocytes (Figures 4-6). 
It can be speculated that the mechanism of action of $\mathrm{mAb} 146 \mathrm{~B} 7$ in the psoriasis xenograft model (Figures 4-6) involves inhibition of a number of reported effects of IL-15: blockade of T cell proliferation, inflammatory cell recruitment, cytokine generation (i.e., TNF- $\alpha$ ), and blockade of the anti-apoptotic activity of IL-15. This is also supported by in vitro data where $\mathrm{mAb} 146 \mathrm{~B} 7$ inhibited IL-15-induced T cell proliferation (Figure $1 \mathrm{c}$ ) and TNF- $\alpha$ production by mononuclear cells (Figure $1 \mathrm{~d}$ ) and reversed IL-15-mediated protection against apoptosis of monocytes (Figure 2).

We propose that the biological characteristics of antibody $146 \mathrm{~B} 7$ are of major importance in obtaining these therapeutic results on the psoriatic xenograft tissue. The IL-15R present on T cells consists of a unique $\alpha$-chain, IL-15R $\alpha$, which has a very high affinity for IL-15 $\left(K_{D} 10^{-11} \mathrm{M}\right)$ and shares a $\beta$-chain and $\gamma$-chain with the $\operatorname{IL}-2 \mathrm{R}(2,36)$. The $\alpha$-chain is crucial for binding of IL-15, whereas the $\beta$ - and $\gamma$-chains are essential for signaling processes. We generated two types of anti-IL-15 antibodies, both of which inhibited IL-15-induced biological effects via different modes of action. Antibody 404E4 represents a classical inhibitory antibody that competes with IL-15R $\alpha$ for binding to IL-15 (Figure 1a). This antibody showed to be the least effective in inhibiting IL-15-induced effects (Figure 2a). Antibody 146B7, however, works via a different mode of action and potently inhibits IL-15-mediated activities without interfering with IL-15-IL-15R $\alpha$ binding (Figure 1a and $b$ ). This antibody binds to IL-15 when it is bound to the receptor and appears to efficiently block biological activity (i.e., inhibition of IL-15-induced T cell proliferation [Figure 1c] and TNF- $\alpha$ production [Figure 1d]) by interfering with the assembly of the IL-15R $\alpha, \beta$, and $\gamma$ signaling components (Figure $3 \mathrm{a}$ ).

The difference between $\mathrm{mAb} 146 \mathrm{~B} 7$ and 404E4 IC50 values observed in proliferation inhibition experiments, taking into account that their affinity for IL-15 is comparable (Figure 1c), are consistent with their suggested modes of action. IL-15 binds to the IL-15R $\alpha$ chain with high affinity $\left(\mathrm{K}_{\mathrm{D}} 10^{-11} \mathrm{M}\right)$, and a receptorblocking antibody, like 404E4, thus needs to exhibit a high affinity, or be used at very high concentrations to be effective. Antibody $146 \mathrm{~B} 7$ may act more potently, as it does not need to compete with IL-15 for IL-15R $\alpha$ binding and solely needs to interfere with the IL-15R $\alpha-\gamma$ assembly. This hypothesis was supported by experiments where mAb $146 \mathrm{~B} 7$ was shown to be much more efficacious in inhibiting IL-15-induced effects (Figure 1c). In this experiment, the antibodies were preincubated with IL-15 before they were added to cells. In another type of experiment in which IL-15 was added to cells prior to addition of antibodies, mAb 404E4 did not block IL-15-induced proliferation in concentrations up to $10 \mu \mathrm{g} / \mathrm{ml}$, whereas $\mathrm{mAb}$ 146B7 inhibited efficiently with a similar IC50 (3.1 0.9 $\mathrm{nM})$ as in experiments where mAb $146 \mathrm{~B} 7$ was preincubated with IL-15.
A recent paper from Dubois et al. (40) provided new insight in the mechanism of action of IL-15. This paper elegantly shows that formation of IL-15/IL-15R $\alpha$ complexes on cell surfaces enables transendosomal recycling of IL-15, leading to persistence of surfacebound IL-15 (Figure 7a). The high affinity of IL-15R $\alpha$ for IL-15, combined with the observation that this cytokine is hard to detect in bodily fluids (40), suggests that most IL-15 in the body is IL-15R $\alpha$ bound. The Dubois et al. (40) paper showed that IL-15/ IL-15R $\alpha$ complexes are capable of induction of transsignaling on cells expressing the IL-15R $\beta$ and $\gamma$-chains (Figure 7c). An antibody like mAb 146B7, blocking signaling via the $\gamma$-chain, would be expected to effectively inhibit signaling in cis as well as in trans (to neighboring $\beta$ - and $\gamma$-chain-expressing cells [Figure $7 \mathrm{~d}$ and e]). In addition, it has been suggested that membrane-bound IL-15 not complexed with IL-15R $\alpha$, and therefore insensitive to acid elution at $\mathrm{pH} 4.4$, exists $(31,41,42)$. There has been uncertainty as to how this form of surface-expressed IL-15 is attached to the membrane. To study whether mAb $146 \mathrm{~B} 7$ binds to acid-elution-insensitive, membrane-bound IL-15, we performed flow cytometry with mAb $146 \mathrm{~B} 7$ and 404E4 on LPS- and IFN- $\gamma$-stimulated monocytes, either treated or untreated with acetate buffer pH 4.4 (not shown). Both acid-treated and -untreated monocytes stained similarly with mAb 146B7, indicating that this antibody recognizes membrane IL-15. Interestingly, however, no surface staining was observed with mAb 404E4, whereas this antibody strongly stained LPS- and IFN- $\gamma$-stimulated monocytes following permeabilization. Because $\mathrm{mAb}$ $404 \mathrm{E} 4$ binds to the IL-15R $\alpha$ binding site on IL-15, this result strongly suggests that membrane IL-15 as defined by Bulfone-Paus et al. (41) is complexed with IL-15R, and treatment at $\mathrm{pH} 4.4$ cannot break this interaction. This is furthermore consistent with observations by Dubois et al. (40) discussed above (Figure 7a) that IL-15/IL-15R $\alpha$ complexes are resistant to transendosomal cycling.

In this article, by using a human antibody against IL-15, we collected evidence for a prominent role of IL-15 in the pathogenesis of psoriasis. An early role of IL-15 in the pathogenesis of inflammatory diseases like rheumatoid arthritis was first postulated by McInnes et al. $(4,5)$. The production of IL- 15 by macrophages in the synovium was suggested to recruit $\mathrm{T}$ cells, leading to the production of other inflammatory cytokines like TNF- $\alpha$. Our in vitro and in vivo studies indicate that $\mathrm{mAb} 146 \mathrm{~B} 7$ is able to inhibit IL-15-induced $\mathrm{T}$ cell proliferation, inhibit production of IL-15-induced TNF- $\alpha$ production, reverse IL-15-mediated protection against apoptosis of monocytes, and decrease the number of inflammatory cells in the psoriatic lesion. As it was suggested for rheumatoid arthritis, IL-15 may, therefore, also play an early role in the in vivo mechanism of the pathogenesis of psoriasis. 


\section{Acknowledgments}

The authors of this article would like to thank Wim Bleeker, Joost Bakker, Eefje Melgert, Marielle Pluyter, Margit Bæksted, Jette Pedersen, and Pernille Albrectsen for their enthusiastic participation in this project and Ingrid Sofie Harbo for statistical analysis of the experimental data.

1. Kirman, I., Vainer, B., and Nielsen, O.H. 1998. Interleukin-15 and its role in chronic inflammatory diseases. Inflamm. Res. 47:285-289.

2. Waldmann, T.A., and Tagaya, Y. 1999. The multifaceted regulation of interleukin-15 expression and the role of this cytokine in NK cell differentiation and host response to intracellular pathogens. Annu. Rev. Immunol. 17:19-49.

3. Fehniger, T.A., and Caligiuri, M.A. 2001. Interleukin 15: biology and relevance to human disease. Blood. 97:14-32.

4. McInnes, I.B., Leung, B.P., Sturrock, R.D., Field, M., and Liew, F.Y. 1997. Interleukin-15 mediates $T$ cell-dependent regulation of tumor necrosis factor-alpha production in rheumatoid arthritis. Nat. Med. 3:189-195.

5. McInnes, I.B., and Liew, F.Y. 1998. Interleukin 15: a proinflammatory role in rheumatoid arthritis synovitis. Immunol. Today. 19:75-79.

6. Grabstein, K.H., et al. 1994. Cloning of a T cell growth factor that interacts with the beta chain of the interleukin-2 receptor. Science. 264:965-968.

7. Musso, T., et al. 1998. Interleukin-15 activates proinflammatory and antimicrobial functions in polymorphonuclear cells. Infect. Immun. 66:2640-2647

8. Angiolillo, A.L., Kanegane, H., Sgadari, C., Reaman, G.H., and Tosato, G. 1997. Interleukin-15 promotes angiogenesis in vivo. Biochem. Biophys. Res. Commun. 233:231-237.

9. Mrozek, E., Anderson, P., and Caligiuri, M.A. 1996. Role of interleukin15 in the development of human CD56+ natural killer cells from CD34+ hematopoietic progenitor cells. Blood. 87:2632-2640.

10. Carson, W.E., et al. 1997. A potential role for interleukin-15 in the regulation of human natural killer cell survival. J. Clin. Invest. 99:937-943.

11. Carson, W.E., et al. 1994. Interleukin (IL) 15 is a novel cytokine that activates human natural killer cells via components of the IL-2 receptor. J. Exp. Med. 180:1395-1403.

12. Ruckert, R., et al. 2000. Inhibition of keratinocyte apoptosis by IL-15 a new parameter in the pathogenesis of psoriasis? J. Immunol. 165:2240-2250

13. Bulfone-Paus, S., et al. 1997. Interleukin-15 protects from lethal apoptosis in vivo. Nat. Med. 3:1124-1128

14. McInnes, I.B., et al. 1996. The role of interleukin-15 in T-cell migration and activation in rheumatoid arthritis. Nat. Med. 2:175-182.

15. Wrone-Smith, T., et al. 1995. Discordant expression of Bcl-x and Bcl-2 by keratinocytes in vitro and psoriatic keratinocytes in vivo. Am. J. Pathol. 146:1079-1088

16. Wrone-Smith, T., et al. 1997. Keratinocytes derived from psoriatic plaques are resistant to apoptosis compared with normal skin. Am. J. Pathol. 151:1321-1329.

17. Nickoloff, B.J. 2001. Creation of psoriatic plaques: the ultimate tumor suppressor pathway. A new model for an ancient T-cell mediated skin disease. J. Cutan. Pathol. 28:57-94.

18. Baadsgaard, O., et al. 1990. UM4D4+ (CDw60) T cells are compartmentalized into psoriatic skin and release lymphokines that induce a keratinocyte phenotype expressed in psoriatic lesions. J. Invest. Dermatol. 95:275-282.

19. Strange, P., et al. 1993. T-lymphocyte clones initiated from lesional psoriatic skin release growth factors that induce keratinocyte proliferation. J. Invest. Dermatol. 101:695-700.
20. Gottlieb, S.L., et al. 1995. Response of psoriasis to a lymphocyte-selective toxin (DAB389IL-2) suggests a primary immune, but not keratinocyte, pathogenic basis. Nat. Med. 1:442-447.

21. Bagel, J., et al. 1998. Administration of DAB389IL-2 to patients with recalcitrant psoriasis: a double-blind, phase II multicenter trial. J. Am Acad. Dermatol. 38:938-944.

22. Schlaak, J.F., et al. 1994. T cells involved in psoriasis vulgaris belong to the Th1 subset. J. Invest. Dermatol. 102:145-149.

23. Uyemura, K., Yamamura, M., Fivenson, D.F., Modlin, R.L., and Nickoloff, B.J. 1993. The cytokine network in lesional and lesion-free psoriatic skin is characterized by a T-helper type 1 cell-mediated response. J. Invest. Dermatol. 101:701-705.

24. Nickoloff, B.J., and Wrone-Smith, T. 1999. Injection of pre-psoriatic skin with CD4+ T cells induces psoriasis. Am. J. Pathol. 155:145-158.

25. Gilhar, A., et al. 2002. Psoriasis is mediated by a cutaneous defect triggered by activated immunocytes: induction of psoriasis by cells with natural killer receptors. J. Invest. Dermatol. 119:384-391.

26. Nickoloff, B.J., Bonish, B., Huang, B.B., and Porcelli, S.A. 2000. Characterization of a $\mathrm{T}$ cell line bearing natural killer receptors and capable of creating psoriasis in a SCID mouse model system. J. Dermatol. Sci. 24:212-225.

27. Ettehadi, P., Greaves, M.W., Wallach, D., Aderka, D., and Camp, R.D. 1994. Elevated tumour necrosis factor-alpha (TNF-alpha) biological activity in psoriatic skin lesions. Clin. Exp. Immunol. 96:146-151.

28. Chaudhari, U., et al. 2001. Efficacy and safety of infliximab monotherapy for plaque-type psoriasis: a randomised trial. Lancet. 357:1842-1847.

29. Mease, P.J., et al. 2000. Etanercept in the treatment of psoriatic arthritis and psoriasis: a randomised trial. Lancet. 356:385-390.

30. Nickoloff, B.J. 2000. Characterization of lymphocyte-dependent angiogenesis using a SCID mouse: human skin model of psoriasis. J. Investig. Dermatol. Symp. Proc. 5:67-73.

31. Ong, P.Y., et al. 2002. Decreased IL-15 may contribute to elevated IgE and acute inflammation in atopic dermatitis. J. Immunol. 168:505-510.

32. Fishwild, D.M., et al. 1996. High-avidity human IgG kappa monoclonal antibodies from a novel strain of minilocus transgenic mice. Nat. Biotechnol. 14:845-851.

33. Kohler, G., and Milstein, C. 1975. Continuous cultures of fused cells secreting antibody of predefined specificity. Nature. 256:495-497.

34. Pettit, D.K., et al. 1997. Structure-function studies of interleukin 15 using site-specific mutagenesis, polyethylene glycol conjugation, and homology modeling. J. Biol. Chem. 272:2312-2318.

35. Dam, T.N., Kang, S., Nickoloff, B.J., and Voorhees, J.J. 1999. 1alpha,25dihydroxycholecalciferol and cyclosporine suppress induction and promote resolution of psoriasis in human skin grafts transplanted on to SCID mice. J. Invest. Dermatol. 113:1082-1089.

36. Waldmann, T., Tagaya, Y., and Bamford, R. 1998. Interleukin-2, interleukin-15, and their receptors. Int. Rev. Immunol. 16:205-226.

37. Bulanova, E., et al. 2001. The IL-15R alpha chain signals through association with Syk in human B cells. J. Immunol. 167:6292-6302.

38. Nickoloff, B.J., Kunkel, S.L., Burdick, M., and Strieter, R.M. 1995. Severe combined immunodeficiency mouse and human psoriatic skin chimeras. Validation of a new animal model. Am. J. Pathol. 146:580-588.

39. Schon, M.P. 1999. Animal models of psoriasis - what can we learn from them? J. Invest. Dermatol. 112:405-410.

40. Dubois, S., Mariner, J., Waldmann, T.A., and Tagaya, Y. 2002. IL-15Ralpha recycles and presents IL-15 In trans to neighboring cells. Immunity. 17:537-547.

41. Musso, T., et al. 1999. Human monocytes constitutively express membrane-bound, biologically active, and interferon-gamma-upregulated interleukin-15. Blood. 93:3531-3539.

42. Rappl, G., et al. 2001. Dermal fibroblasts sustain proliferation of activated $t$ cells via membrane-bound interleukin-15 upon long-term stimulation with tumor necrosis factor alpha. J. Invest. Immunol. 116:102-109. 